\title{
Causal-Consistent Reversibility in a Tuple-Based Language
}

\author{
Elena Giachino \\ and Ivan Lanese \\ Focus Team, University of Bologna/INRIA, Italy \\ Email: elena.giachino@unibo.it, ivan.lanese@gmail.com
}

\author{
Claudio Antares Mezzina \\ SOA Unit \\ FBK Trento, Italy \\ Email: mezzina@fbk.eu
}

\author{
Francesco Tiezzi \\ School of Science and Technology \\ University of Camerino, Italy \\ Email: francesco.tiezzi@unicam.it
}

\begin{abstract}
Causal-consistent reversibility is a natural way of undoing concurrent computations. We study causal-consistent reversibility in the context of $\mu$ KLAIM, a formal coordination language based on distributed tuple spaces. We consider both uncontrolled reversibility, suitable to study the basic properties of the reversibility mechanism, and controlled reversibility based on a rollback operator, more suitable for programming applications. The causality structure of the language, and thus the definition of its reversible semantics, differs from all the reversible languages in the literature because of its generative communication paradigm. In particular, the reversible behavior of $\mu$ KLAIM read primitive, reading a tuple without consuming it, cannot be matched using channel-based communication. We illustrate the reversible extensions of $\mu$ KLAIM on a simple, but realistic, application scenario.
\end{abstract}

\section{INTRODUCTION}

Reversibility is a main ingredient of different kinds of systems, including, e.g., biological systems or quantum systems. We are mainly interested in reversibility as a support for programming reliable concurrent systems. The basic idea is that if a system reaches an undesired state (e.g., an error or deadlock state), reversibility can be used to go back to a past desirable state. Our claim is that the ability to reverse actions is key to understanding and improving existing patterns for programming reliable systems, such as transactions or checkpointing, and to devise new ones.

Studying reversibility in a concurrent setting is particularly tricky. In fact, even the definition of reversibility is different w.r.t. the sequential one, since "recursively undo the last action" is not meaningful in a concurrent scenario, where many actions can be executed at the same time by different threads. This observation led to the concept of causalconsistent reversibility: one may undo any action if no other action depending on it has been executed (and not undone). Building on this definition, reversible extensions of many concurrent calculi and languages have been defined, e.g., for CCS [5], [18], $\pi$-calculus [4], higher-order $\pi$ [14] and $\mu \mathrm{Oz}$ [17]. However, to figure out how to make a general programming language reversible, the interplay between reversibility and many common language features has still to be understood. In particular, none of the reversible calculi in the literature features tuple-based communication: they all consider channel-based communication.

This paper studies reversibility in the context of $\mu$ KLAIM [11], a formal language based on distributed tuple spaces derived from the coordination language KLAIM [8]. $\mu$ KLAIM contrasts on two main points with all the languages whose causal-consistent reversible semantics has been studied in the literature. First, it features localities. Second, it uses tuple-based communication as the interaction paradigm, supported by five primitives. Primitives out and in respectively insert tuples into and remove them from tuple spaces. Primitives eval, to execute a process on a possibly remote location, and newloc, creating a new location, support distribution. Finally, $\mu$ KLAIM features the primitive read, which reads a tuple without consuming it. This last primitive allows concurrent processes to access a shared resource while staying independent, thus undoing the actions of one of them has no impact on the others. This behavior, common when manipulating shared data structures, e.g. in software transactional memories, cannot be programmed using only in and out primitives, nor using channel-based communications, since the resulting causal structure would be different.

In this paper, we first study uncontrolled reversibility (Section III), i.e. we define how a process executes forward or backward, but not when it is supposed to do so. This produces a clean algebraic setting, suitable to prove properties of our reversibility mechanism. In particular, we show that reversible $\mu$ KLAIM ( $\mu$ KLAIM for short) is causally consistent (Theorem 1), and that its forward computations correspond to $\mu$ KLAIM computations (Lemmas 2 and 3). However, uncontrolled reversibility is not suitable for programming error recovery activities. In fact, it does not provide a mechanism to trigger a backward computation in case of error: backward actions are always enabled. Even more, a R $\mu$ KLAIM process may always diverge by doing and undoing the same action forever.

To solve this problem, we build on top of R $\mu$ KLAIM a language with controlled reversibility, CR $\mu$ KLAIM (Section IV). CR $\mu$ KLAIM computation normally proceeds forward, but the programmer may ask for a rollback using a dedicated roll operator. This operator undoes a given past action, and all its consequences, but it does not affect independent actions. The roll operator is based on the uncontrolled reversibility mechanism, but it is much more suitable to exploit reversibility for programming actual applications. We put CR $\mu$ KLAIM at work on a practical example about franchising (Section V). Proofs and additional examples are available in the companion technical report [10].

From the practical perspective, we believe that the formal approach proposed in this paper is a further step towards the sound development of a real-world reversible language for 


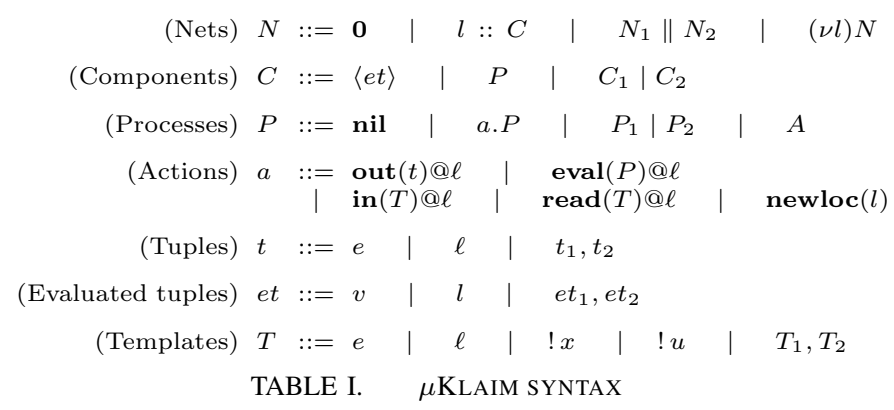

(Monoid) $\quad N\left\|\mathbf{0} \equiv N \quad N_{1}\right\| N_{2} \equiv N_{2} \| N_{1}$ $\left(N_{1} \| N_{2}\right)\left\|N_{3} \equiv N_{1}\right\|\left(N_{2} \| N_{3}\right)$

(RCom) $\quad\left(\nu l_{1}\right)\left(\nu l_{2}\right) N \equiv\left(\nu l_{2}\right)\left(\nu l_{1}\right) N$

(PDef) $\quad l:: A \equiv l:: P$ if $A \triangleq P$

(Ext) $\quad N_{1} \|(\nu l) N_{2} \equiv(\nu l)\left(N_{1} \| N_{2}\right)$ if $l \notin f n\left(N_{1}\right)$

(Alpha) $\quad N \equiv N^{\prime}$ if $N={ }_{\alpha} N^{\prime}$

(Abs) $\quad l:: C \equiv l::(C \mid \mathbf{n i l})$

(Clone) $\quad l:: C_{1} \mid C_{2} \equiv l:: C_{1} \| l:: C_{2}$

TABLE II. $\quad \mu$ KLAIM STRUCTURAL CONGRUENCE

programming distributed systems. Its main benefit with respect to traditional languages would be to relieve the programmer from coding rollback activities from scratch: they can be easily obtained by applying the rollback operator.

\section{II. $\mu$ KLAIM SYNTAX AND SEMANTICS}

KLAIM [8] is a formal coordination language designed to provide programmers with primitives for handling physical distribution, scoping and mobility of processes. KLAIM is based on the Linda [9] generative communication paradigm. Communication in KLAIM is achieved by sharing distributed tuple spaces, where processes insert, read and withdraw tuples. The data retrieving mechanism is based on associative patternmatching. In this paper, to simplify the presentation, we consider a core language of KLAIM, called $\mu$ KLAIM. We refer to [2] for a detailed account of KLAIM and $\mu$ KLAIM.

Syntax. The syntax of $\mu$ KLAIM is in Table I. We assume four disjoint sets: the set of localities, ranged over by $l$, of locality variables, ranged over by $u$, of value variables, ranged over by $x$, and of process identifiers, ranged over by $A$. Localities are the addresses (i.e., network references) of nodes and are the syntactic ingredient used to model administrative domains. In $\mu$ KLAIM, communicable objects are (evaluated) tuples, i.e., sequences of actual fields. Tuple fields may contain expressions, localities or locality variables. We leave the expressions e unspecified; we just assume they include values $(v)$ and value variables. Names, i.e., locality variables and localities, are ranged over by $\ell$. We assume each process identifier $A$ has a single definition $A \triangleq P$, available at any locality of the net.

Nets are finite plain collections of nodes where components, i.e., processes and evaluated tuples, can be hosted. A node is a pair $l:: C$, where $l$ is the address of the node and
$C$ is the hosted component. In the net $(\nu l) N$, the scope of the name $l$ is restricted to $N$. 0 denotes the empty net.

Processes, the $\mu$ KLAIM active computational units, may be inactive as nil, prefixed by an action as a.P, parallel compositions as $P_{1} \mid P_{2}$, and process identifiers as $A$. We may drop trailing nils. Processes may be executed concurrently either at the same locality or at different localities and can perform actions.

Actions out, in and read add/withdraw/access data from repositories. Action eval activates a new thread of execution in a (possibly remote) node, and newloc creates new nodes. All actions but newloc indicate their target locality. Actions in and read are blocking and use, as patterns to select data in repositories, templates: sequences of actual and formal fields, where the latter are written $! x$ and $! u$, and are used to bind value variables to values and locality variables to localities, respectively.

Localities and variables can be bound inside processes and nets: newloc $(l) . P$ binds name $l$ in $P$, and $(\nu l) N$ binds $l$ in $N$. Prefixes in $(\ldots, !, \ldots) @ \ell . P$ and $\operatorname{read}(\ldots, !, \ldots) @ \ell . P$ bind variable - in $P$. A locality/variable that is not bound is called free. The set $f n(\cdot)$ of free names of a term is defined accordingly. As usual, we say that two terms are $\alpha$-equivalent, written $={ }_{\alpha}$, if one can be obtained from the other by consistently renaming bound localities/variables. In the sequel, we assume Barendregt convention, i.e. we work only with terms whose bound variables and bound localities are all distinct and different from the free ones.

Operational semantics. The operational semantics of $\mu$ KLAIM is given in terms of a structural congruence relation and a reduction relation expressing the evolution of a net. The structural congruence $\equiv$ is defined as the least congruence closed under the equational laws in Table II. Law (Abs) states that nil is the identity for $\cdot \mid \cdot$. Law (Clone) turns a parallel between co-located components into a parallel between nodes (thus, it is also used, together with (Monoid) laws, to achieve commutativity and associativity of $\cdot \mid \cdot)$. The other laws are standard.

We define the auxiliary pattern-matching function $\operatorname{match}(\cdot, \cdot)$ as the smallest function closed under the rules in Table III. Intuitively, a tuple matches a template if they have the same number of fields, and corresponding fields match: two values/localities match only if they are identical, bound value/locality variables match any value/locality, and the matching for free variables always fails. When match $(T, t)$ succeeds, it returns a substitution for the variables in $T$; otherwise, it is undefined. A substitution $\sigma$ is a function with finite domain from variables to localities/values, and is written as a collection of pairs of the form $v / x$ or $l / u$. We use $\circ$ to denote substitution composition and $\epsilon$ to denote the empty substitution.

We assume the existence of a function $\llbracket \cdot \rrbracket$ for evaluating tuples and templates, which computes the value of closed expressions occurring in a tuple/template. Its definition depends on the definition of expressions, which we left unspecified. Only evaluated tuples $\langle e t\rangle$ are stored in tuple spaces.

We say a relation $\mathcal{R}$ is evaluation closed if it is closed under active contexts, i.e. $N_{1} \mathcal{R} N_{1}^{\prime}$ implies $\left(N_{1} \| N_{2}\right) \mathcal{R}\left(N_{1}^{\prime} \|\right.$ 


$$
\begin{gathered}
\operatorname{match}\left(T_{1}, t_{1}\right)=\sigma_{1} \quad \operatorname{match}\left(T_{2}, t_{2}\right)=\sigma_{2} \\
\operatorname{match}\left(\left(T_{1}, T_{2}\right),\left(t_{1}, t_{2}\right)\right)=\sigma_{1} \circ \sigma_{2} \\
\operatorname{match}(v, v)=\epsilon \quad \operatorname{match}(! x, v)=[v / x] \\
\operatorname{match}(l, l)=\epsilon \quad \operatorname{match}(! u, l)=[l / u]
\end{gathered}
$$

TABLE III. $\quad \mu$ KLAIM MATCHING RULES

$$
\begin{aligned}
& \frac{\llbracket t \rrbracket=e t}{l:: \operatorname{out}(t) @ l^{\prime} . P \| l^{\prime}:: \text { nil } \mapsto l:: P \| l^{\prime}::\langle e t\rangle}(\text { Out }) \\
& \operatorname{match}(\llbracket T \rrbracket, e t)=\sigma \\
& \overline{l:: \operatorname{in}(T) @ l^{\prime} . P\left\|l^{\prime}::\langle e t\rangle \mapsto l:: P \sigma\right\| l^{\prime}:: \mathbf{n i l}}
\end{aligned}
$$$$
\frac{\operatorname{match}(\llbracket T \rrbracket, e t)=\sigma}{l:: \operatorname{read}(T) @ l^{\prime} . P\left\|l^{\prime}::\langle e t\rangle \mapsto l:: P \sigma\right\| l^{\prime}::\langle e t\rangle}(\text { Read })
$$$$
l:: \operatorname{newloc}\left(l^{\prime}\right) \cdot P \mapsto\left(\nu l^{\prime}\right)\left(l:: P \| l^{\prime}:: \operatorname{nil}\right)(N e w)
$$$$
l:: \operatorname{eval}(Q) @ l^{\prime} . P \| l^{\prime}:: \text { nil } \mapsto l:: P \| l^{\prime}:: Q \text { (Eval) }
$$

TABLE IV. $\quad \mu$ KLAIM OPERATIONAL SEMANTICS

$\left.N_{2}\right)$ and $(\nu l) N_{1} \mathcal{R}(\nu l) N_{1}^{\prime}$, and under structural congruence, i.e. $N \equiv M \mathcal{R} M^{\prime} \equiv N^{\prime}$ implies $N \mathcal{R} N^{\prime}$. The $\mu$ KLAIM reduction relation $\mapsto$ is the smallest evaluation-closed relation satisfying the rules in Table IV. All rules for (possibly remote) actions out, eval, in and read require the existence of the target node $l^{\prime}$. In rule (Out) the action can proceed only if the tuple in its argument is evaluable. If so, the evaluated tuple is released in the target node $l^{\prime}$. Rules (In) and (Read) require the target node to contain a tuple matching their (necessarily evaluable) template argument $T$. The content of the matched tuple is then used to replace the free occurrences of the variables bound by $T$ in $P$, the continuation of the process performing the actions. Action in consumes the matched tuple, while action read does not. Rule (New) creates a new (private) node. Rule (Eval) launches a new thread executing process $Q$ on a target node $l^{\prime}$.

\section{UNCONTROLLED REVERSIBILITY}

In this section we define $\mathrm{R} \mu \mathrm{K}$ LAIM, an extension of $\mu$ KLAIM with uncontrolled reversibility. R $\mu$ KLAIM nets allow both forward actions, modeling $\mu$ KLAIM actions, and backward actions, undoing them, but nothing is specified about whether to prefer forward steps over backward steps, or vice versa. While the general approach follows [14], the technical development is considerably different.

We first present $\mathrm{R} \mu \mathrm{KLAIM}$, with a simple example to point out the peculiarity of the causality relationships produced by $\mathrm{R} \mu$ KLAIM constructs. Then, we show that the typical properties expected from a reversible formalism hold.

Syntax. R $\mu$ KLAim syntax is in Table V. Processes, actions, (evaluated) tuples, and templates are the same as in $\mu$ KLAIM (Table I). The main ingredients of $\mathrm{R} \mu \mathrm{KLAIM}$ are keys $k$, uniquely identifying tuples and processes, memories $\mu$, storing information for undoing past actions, and connectors $k_{1} \prec\left(k_{2}, k_{3}\right)$, tracking causality information. More precisely,

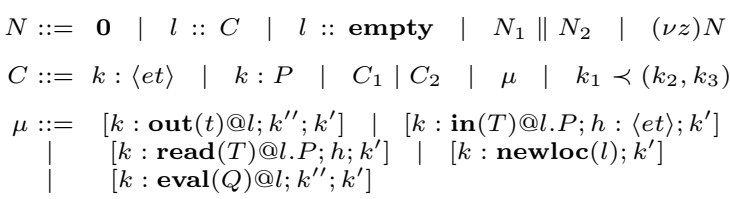

TABLE V. R $\mu$ KLAIM SYNTAX

we have the additional syntactic category of keys, ranged over by $k, h, \ldots$ We use $z$ to range over keys and localities. Uniqueness of keys is enforced by using restriction, the only binder for keys (free and bound keys and $\alpha$-conversion are defined as usual, and from now on $f n(N)$ also includes free keys), and by only considering well-formed nets.

Definition 1 (Initial and well-formed nets): A R $\mu$ KLAIM net is initial if it has no memories, no connectors, and all its keys are distinct. A R $\mu$ KLAIM net is well formed if it can be obtained by forward or backward reductions (cfr. Definition 2) starting from an initial net.

Keys are needed to distinguish processes/tuples with the same form but different histories, thus allowing for different backward actions. Histories are stored in memories and connectors. A memory keeps track of a past action, thus we have one kind of memoryfor each kind of action. All of them store the prefix giving rise to the action and the fresh key $k^{\prime}$ generated for the continuation. Furthermore, memories for in and read store their original continuation $P$, since it cannot be recovered from the running one, obtained by applying a substitution - a non reversible transformation ${ }^{1}$. Also, the out memory stores the key $k^{\prime \prime}$ of the created tuple, the eval's one the key $k^{\prime \prime}$ of the spawned process, and the in's one the consumed tuple $h$ : $\langle e t\rangle$. The memory for read only needs the key $h$ of the read tuple, still available in the term and uniquely identified by key $h$. Connector $k_{1} \prec\left(k_{2}, k_{3}\right)$ recalls that processes with keys $k_{2}$ and $k_{3}$ originated from the split of process tagged by $k_{1}$. Finally, we distinguish empty localities, $l::$ empty, containing no information, from localities $l:: k$ : nil containing a nil process with its key $k$, which may interact with a memory to perform a backward action.

Operational semantics. Structural congruence for $\mathrm{R} \mu$ KLAIM extends the one for $\mu$ KLAIM in Table II to deal with keys: new rules (Garb) and (Split) and updated rules are reported in Table VI. Rule (Garb) garbage-collects unused keys. Rule (Split) splits parallel processes using a connector and generating fresh keys to preserve keys uniqueness.

Definition 2 ( $\mathrm{R} \mu \mathrm{KLAIM}$ semantics): The operational semantics of $\mathrm{R} \mu \mathrm{KLAIM}$ consists of a forward reduction relation $\mapsto_{r}$ and a backward reduction relation $\rightsquigarrow_{r}$. They are the smallest evaluation-closed relations (now closure under active contexts considers also restriction on keys) satisfying the rules in Table VII.

Forward rules correspond to $\mu$ KLAIM rules, adding the management of keys and memories. We have one backward rule for each forward rule, undoing the forward action. Consider rule (Out). Existence of the target node $l^{\prime}$ is guaranteed by

\footnotetext{
${ }^{1}$ One may look for more compact ways to store history information. This issue is considered for reversible $\mu \mathrm{Oz}$ in [17], but it is out of the scope of the present paper.
} 

(RCom) $\left(\nu z_{1}\right)\left(\nu z_{2}\right) N \equiv\left(\nu z_{2}\right)\left(\nu z_{1}\right) N$
(PDef) $l:: k: A \equiv l:: k: P \quad$ if $A \triangleq P$
(Ext) $N_{1} \|(\nu z) N_{2} \equiv(\nu z)\left(N_{1} \| N_{2}\right)$ if $z \notin f n\left(N_{1}\right)$
(Abs) $l:: C \equiv l:: C \| l::$ empty
$(G a r b)(\nu k) \mathbf{0} \equiv \mathbf{0}$
(Split) $l:: k: P\left|Q \equiv\left(\nu k_{1}, k_{2}\right) l:: k \prec\left(k_{1}, k_{2}\right)\right| k_{1}: P \mid k_{2}: Q$

TABLE VI. R $\mu$ KLAIM STRUCTURAL CONGRUENCE

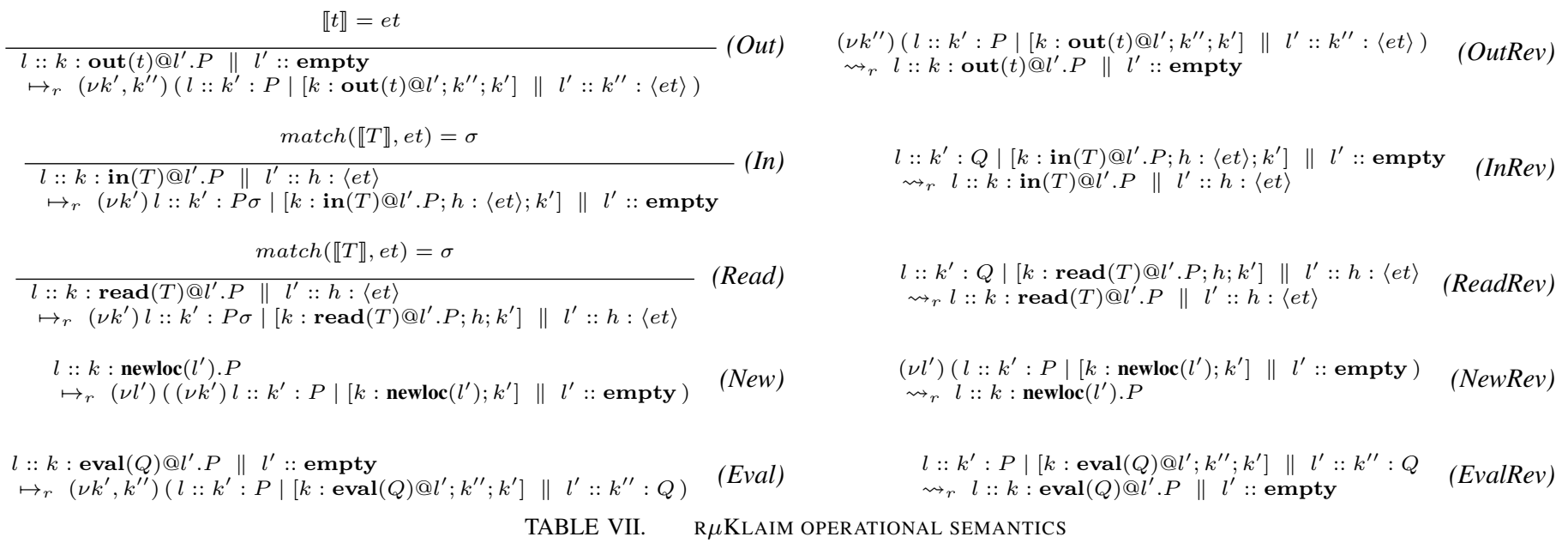

requiring a parallel term $l^{\prime}::$ empty. If locality $l^{\prime}$ is not empty, such term can be generated by structural rule (Abs). Two fresh keys $k^{\prime}$ and $k^{\prime \prime}$ are created to tag the continuation $P$ and the new tuple $\langle e t\rangle$, respectively. Also, a memory is created (in the locality where the out prefix was) storing all the relevant information. The corresponding backward rule, (OutRev), may trigger if a memory for out with continuation key $k^{\prime}$ and with created tuple key $k^{\prime \prime}$ finds a process with key $k^{\prime}$ in the same locality and a tuple with key $k^{\prime \prime}$ in the target locality $l^{\prime}$. Requiring that $l^{\prime}$ contains only the tuple tagged by $k^{\prime \prime}$ is not restrictive, thanks to structural rule (Clone). Note also that all the actions performed by the continuation process $k^{\prime}$ : $P$ have to be undone beforehand, otherwise no process with key $k^{\prime}$ would be available at top level (i.e., outside memories). Moreover, the tuple generated by the out, which will be removed by the backward reduction, must bear key $k^{\prime \prime}$ as when it was generated. Note the restriction on key $k^{\prime \prime}:$ this is needed to ensure that all the occurrences of $k^{\prime \prime}$ are inside the term, i.e. $k^{\prime \prime}$ occurs only in the out memory and in the tuple. This ensures that read actions that have accessed the tuple, whose resulting memory would contain $k^{\prime \prime}$, have been undone. The problem of read dependencies is peculiar to the $\mu$ KLAIM setting, and it does not emerge in the other works in the reversibility literature. On the other hand, restricting key $k^{\prime}$ in rule (OutRev) would be redundant since in a well-formed net it can occur only twice, and both the occurrences are consumed by the rule. Thus, the restriction can be garbage collected by using structural congruence. Executing the backward rule (OutRev) undoes the effect of the forward rule (Out), as proved by the Loop lemma below. In rule (Eval), $k^{\prime \prime}$ labels the spawned process $Q$. No restriction on $k^{\prime \prime}$ is required in rule (EvalRev), since $k^{\prime \prime}$ cannot occur elsewhere in the term. In rule (In) the consumed tuple is stored in the memory, while in rule (Read) only the key is needed since the tuple is still in the term, and its key is unchanged. Rule (New) creates a new, empty locality. In rule (NewRev) we again use restriction (now on the name $l^{\prime}$ of the locality) to ensure that no other locality with the same name exists. This could be possible since localities may be split using structural congruence rules (Abs) or (Clone).

Example 1: We show an example to clarify the difference between the behavior of a $\mathrm{R} \mu \mathrm{KLAIM}$ read action and its implementations in the other reversible languages we are aware of. They feature channel-based communication, thus the only way of accessing a resource is consuming it with an input and restoring it with an output. This corresponds to the behavior we obtain in R $\mu$ KLAIM by using an in followed by an out. Consider a $\mathrm{R} \mu \mathrm{KLAIM}$ net $N$ with three nodes, $l_{1}$ hosting a tuple $\langle f o o\rangle$, and $l_{2}$ and $l_{3}$ hosting processes willing to access such tuple:

$$
\begin{aligned}
& N^{\prime}=l_{1}:: k_{1}:\langle\text { foo }\rangle \| l_{2}:: k_{2}: \operatorname{in}(f o o) @ l_{1} . \text { out }(f o o) @ l_{1} . P \\
& \| l_{3}:: k_{3}: \operatorname{in}(f o o) @ l_{1} \text {.out }(f \circ o) @ l_{1} . P^{\prime}
\end{aligned}
$$

By executing first the sequence of in and out in $l_{2}$, and then the corresponding sequence in $l_{3}$ (the order is relevant), the net evolves to:

$$
\begin{aligned}
& \left(\nu k_{2}^{\prime}, k_{2}^{\prime \prime}, k_{2}^{\prime \prime \prime}, k_{3}^{\prime}, k_{3}^{\prime \prime}, k_{3}^{\prime \prime \prime}\right)\left(l_{1}:: k_{3}^{\prime \prime \prime}:\langle\text { foo }\rangle\right. \\
& \| l_{2}:: k_{2}^{\prime \prime}: P \mid\left[k_{2}: \operatorname{in}(\text { foo }) @ l_{1} \cdot \operatorname{out}(\text { foo }) @ l_{1} . P ; k_{1}:\langle\text { foo }\rangle ; k_{2}^{\prime}\right] \\
& \mid \begin{array}{rll}
\mid & {\left[k_{2}^{\prime}: \operatorname{out}(\text { foo }) @ l_{1} ; k_{2}^{\prime \prime \prime} ; k_{2}^{\prime \prime}\right]}
\end{array} \\
& \| l_{3}:: k_{3}^{\prime \prime}: P^{\prime} \mid\left[k_{3}: \operatorname{in}(\text { foo }) @ l_{1} \cdot \operatorname{out}(\text { foo }) @ l_{1} . P^{\prime} ; k_{2}^{\prime \prime \prime}:\langle\text { foo }\rangle ; k_{3}^{\prime}\right] \\
& \left.\mid\left[k_{3}^{\prime}: \operatorname{out}(\text { foo }) @ l_{1} ; k_{3}^{\prime \prime \prime} ; k_{3}^{\prime \prime}\right]\right)
\end{aligned}
$$

Now, the process in $l_{2}$ cannot immediately perform a backward step, since it needs the tuple $k_{2}^{\prime \prime \prime}:\langle f o o\rangle$ in $l_{1}$, while only $k_{3}^{\prime \prime \prime}:\langle f o o\rangle$ is available. The former tuple has been consumed by the in action at $l_{3}$ (see the corresponding memory stored in $l_{3}$ ) and then replaced by the latter by the out action at $l_{3}$. This means that to perform the backward step of the process in $l_{2}$ one needs first to reverse the process in $l_{3}$. Of course, this is not desired when the processes are accessing a shared resource in read-only modality. This is nevertheless the behavior obtained if the resource is, e.g., a message in $\rho \pi$ [14] or an output process in [5], [18], [4].

The problem can be solved in $\mathrm{R} \mu \mathrm{KLAIM}$ using the read primitive. Let us replace in the net above each sequence of in and out with a read:

$$
\begin{aligned}
& N=l_{1}:: k_{1}:\langle f o o\rangle \| l_{2}:: k_{2}: \operatorname{read}(f o o) @ l_{1} . P \\
& \| l_{3}:: k_{3}: \operatorname{read}(\text { foo }) @ l_{1} . P^{\prime}
\end{aligned}
$$




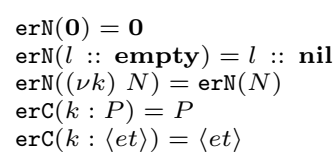

TABLE VIII.
$\operatorname{erN}\left(N_{1} \| N_{2}\right)=\operatorname{erN}\left(N_{1}\right) \| \operatorname{erN}\left(N_{2}\right)$

$\operatorname{erN}(l:: C)=l:: \operatorname{erc}(C)$

$\operatorname{erN}((\nu l) N)=(\nu l) \operatorname{erN}(N)$

$\operatorname{erc}\left(C_{1} \mid C_{2}\right)=\operatorname{erC}\left(C_{1}\right) \mid \operatorname{erC}\left(C_{2}\right)$

$\operatorname{erC}\left(k \prec\left(k_{1}, k_{2}\right)\right)=$ nil $\quad \operatorname{erC}(\mu)=$ nil

erN AND erC FUNCTIONS

By executing the two read actions (the order is now irrelevant), the net $N$ evolves to:

$$
\begin{aligned}
& \left(\nu k_{2}^{\prime}, k_{3}^{\prime}\right)\left(l_{1}:: k_{1}:\langle\text { foo }\rangle\right. \\
& \| l_{2}:: k_{2}^{\prime}: P \mid\left[k_{2}: \operatorname{read}(\text { foo }) @ l_{1} \cdot P ; k_{1} ; k_{2}^{\prime}\right] \\
& \left.\| l_{3}:: k_{3}^{\prime}: P^{\prime} \mid\left[k_{3}: \operatorname{read}(\text { foo }) @ l_{1} . P^{\prime} ; k_{1} ; k_{3}^{\prime}\right]\right)
\end{aligned}
$$

Any of the two processes, say $l_{2}$, can undo the executed read action without affecting the execution of the other one. Thus, applying rule (ReadRev) we get:

$$
\begin{aligned}
& \left(\nu k_{2}^{\prime}, k_{3}^{\prime}\right)\left(l_{1}:: k_{1}:\langle\text { foo }\rangle\right. \\
& \| l_{2}:: k_{2}: \operatorname{read}(\text { foo }) @ l_{1} \cdot P \\
& \left.\| l_{3}:: k_{3}^{\prime}: P^{\prime} \mid\left[k_{3}: \operatorname{read}(\text { foo }) @ l_{1} \cdot P^{\prime} ; k_{1} ; k_{3}^{\prime}\right]\right)
\end{aligned}
$$

Basic properties. We now show that $\mathrm{R} \mu$ KLAIM respects the $\mu$ KLAIM semantics, and that it is causally consistent. We first introduce some auxiliary definitions.

Well-formed nets satisfy the property below, where, given memories of the shape $\left[k: \operatorname{out}(t) @ l ; k^{\prime \prime} ; k^{\prime}\right]$, $\left[k: \operatorname{in}(T) @ l . P ; h^{\prime}:\langle t\rangle ; k^{\prime}\right], \quad\left[k: \operatorname{read}(T) @ l . P ; h ; k^{\prime}\right]$, $\left[k: \operatorname{eval}(Q) @ l ; k^{\prime \prime} ; k^{\prime}\right] \quad$ and $\left[k: \operatorname{newloc}(l) ; k^{\prime}\right], \quad$ and connectors of the shape $k \prec\left(k^{\prime}, k^{\prime \prime}\right)$, we call $k$ the head of those memories/connector, and $k^{\prime}$ and, when they occur, $k^{\prime \prime}$ or $h$, the tail. Keys $h$ and $h^{\prime}$ occur in an input position.

Definition 3 (Complete net): A net $N$ is complete, written complete $(N)$, if: (i) for each key $k$ in the tail of a memory/connector of $N$ there exists in $N$ (possibly inside a memory) either a process $k: P$, or a tuple $k:\langle t\rangle$, or a connector $k \prec\left(h_{1}, h_{2}\right)$ and, unless all the occurrences of $k$ are in input positions, $k$ is bound in $N$; and (ii) for each memory $\left[k: \operatorname{newloc}(l) ; k^{\prime}\right]$ in $N$ there exists in $N$ a node named $l$ and $l$ is bound in $N$.

Lemma 1: For each well-formed net $N$ : (i) all keys occurring in $N$ attached to processes or tuples (possibly in a memory) are distinct, and (ii) $N$ is complete.

From a R $\mu$ KLAIM net we can derive a $\mu$ KLAIM net by removing history and causality information. This is formalized by function erN (and the auxiliary function erC for components) defined in Table VIII. The following lemmas state the correspondence between R $\mu$ KLAIM forward semantics and $\mu$ KLAIM semantics.

Lemma 2: Let $N$ and $M$ be two R $\mu$ KLAIM nets such that $N \mapsto_{r} M$. Then $\operatorname{erN}(N) \mapsto \operatorname{erN}(M)$.

Lemma 3: Let $R$ and $S$ be two $\mu$ KLAIM nets such that $R \mapsto S$. Then for all $\mathrm{R} \mu \mathrm{KLAIM}$ nets $M$ such that $\operatorname{erN}(M)=$ $R$ there exists a R $\mu$ KLAIM net $N$ such that $M \mapsto_{r} N$ and $\operatorname{erN}(N) \equiv S$.

The Loop lemma below shows that each reduction has an inverse.

Lemma 4 (Loop lemma): For all well-formed $\mathrm{R} \mu \mathrm{KLAIM}$ nets $N$ and $M$, the following holds: $N \mapsto_{r} M \Longleftrightarrow M \rightsquigarrow_{r} N$.
We now move to the proof that $\mathrm{R} \mu \mathrm{KLAIM}$ is indeed causally consistent.

In a forward reduction $N \mapsto_{r} M$ we call forward memory the memory $\mu$ which does not occur in $N$ and occurs in $M$. Similarly, in a backward reduction $N \rightsquigarrow_{r} M$ we call backward memory the memory $\mu$ which occurs in $N$ and does not occur in $M$. We call transition a triplet of the form $N \stackrel{\mu_{\mapsto \rightarrow}}{\longrightarrow} M$, or $N \stackrel{\mu_{\rightsquigarrow}}{\longrightarrow} M$, where $N, M$ are well-formed nets, and $\mu$ is the forward/backward memory of the reduction. We call $N$ the source of the transition, $M$ its target. We let $\eta$ range over labels $\mu_{\mapsto_{r}}$ and $\mu_{\rightsquigarrow_{r}}$. If $\eta=\mu_{\mapsto_{r}}$, then $\eta_{\bullet}=\mu_{\rightsquigarrow_{r}}$, and vice versa. Without loss of generality we restrict our attention to transitions derived without using $\alpha$-conversion. We also assume that when structural rule (Split) is applied from left to right creating a connector $h \prec\left(k_{1}, k_{2}\right)$, there is a fixed function determining $k_{1}$ and $k_{2}$ from $h$, and that different values of $h$ produce different values of $k_{1}$ and $k_{2}$. This is needed to avoid that the same name is used with different meanings (cfr. the definition of closure below). Two transitions are coinitial if they have the same source, cofinal if they have the same target, and composable if the target of the first one is the source of the second one. A sequence of pairwise composable transitions is called a trace. We let $\delta$ range over transitions and $\theta$ range over traces. If $\delta$ is a transition then $\delta$. denotes its inverse. Notions of source, target and composability extend naturally to traces. We denote with $\epsilon_{M}$ the empty trace with source $M$, and with $\theta_{1} ; \theta_{2}$ the composition of two composable traces $\theta_{1}$ and $\theta_{2}$. The stamp $\lambda\left(\mu_{\mapsto_{r}}\right)$ of a memory $\mu$ is:

$$
\begin{array}{ll}
\lambda\left(\left[k: \operatorname{out}(t) @ l ; k^{\prime \prime} ; k^{\prime}\right]\right) & =\left\{k, k^{\prime}, k^{\prime \prime}, \mathrm{r}(l)\right\} \\
\lambda\left(\left[k: \operatorname{in}(T) @ l \cdot P ; k^{\prime \prime}:\langle e t\rangle ; k^{\prime}\right]\right) & =\left\{k, k^{\prime}, k^{\prime \prime}, \mathrm{r}(l)\right\} \\
\lambda\left(\left[k: \operatorname{read}(T) @ l . P ; k^{\prime \prime} ; k^{\prime}\right]\right) & =\left\{k, \mathrm{r}\left(k^{\prime \prime}\right), k^{\prime}, \mathrm{r}(l)\right\} \\
\lambda\left(\left[k: \operatorname{eval}(Q) @ l ; k^{\prime \prime} ; k^{\prime}\right]\right) & =\left\{k, k^{\prime}, k^{\prime \prime}, \mathrm{r}(l)\right\} \\
\lambda\left(\left[k: \operatorname{newloc}(l) ; k^{\prime}\right]\right) & =\left\{k, k^{\prime}, l\right\}
\end{array}
$$

We set $\lambda\left(\mu_{\rightsquigarrow_{r}}\right)=\lambda\left(\mu_{\mapsto_{r}}\right)$. The stamp of a memory defines the resources used by the corresponding transitions. The notation $\mathrm{r}(z)$ highlights that resource $z$ is used in read-only modality. All actions but newloc use a locality name in a read-only modality. We use $\kappa$ to range over tags $\mathrm{r}(z)$ and $z$. We define the closure w.r.t. a net $N$ of a tag $\kappa$ as closure $_{N}(\kappa)=\{\kappa\} \cup$ closure $_{N}(h)$ if $\kappa=k_{1}$ or $\kappa=k_{2}$ and $h \prec\left(k_{1}, k_{2}\right)$ occurs in $N,\{\kappa\}$ otherwise. We define the closure over a set $K$ of tags as closure $_{N}(K)=\bigcup_{\kappa \in K}$ closure $_{N}(\kappa)$. The closure captures that a connector $h \prec\left(k_{1}, k_{2}\right)$ means that resources $k_{1}$ and $k_{2}$ are part of resource $h$.

Definition 4 (Concurrent transitions): Two coinitial transitions $M \stackrel{\eta_{1}}{\longrightarrow} N_{1}$ and $M \stackrel{\eta_{2}}{\longrightarrow} N_{2}$ are in conflict if, for some resource $z$, one of the following holds: (i) $z \in$ closure $_{M \| N_{1}}\left(\lambda\left(\eta_{1}\right)\right)$ and $z \in$ closure $_{M \| N_{2}}\left(\lambda\left(\eta_{2}\right)\right)$, (ii) $\mathrm{r}(z) \in \lambda\left(\eta_{1}\right)$ and $z \in$ closure $_{M \| N_{2}}\left(\lambda\left(\eta_{2}\right)\right)$, or (iii) $z \in$ closure $_{M \| N_{1}}\left(\lambda\left(\eta_{1}\right)\right)$ and $r(z) \in \lambda\left(\eta_{2}\right)$. Two coinitial transitions are concurrent if they are not in conflict.

Essentially, two transitions are in conflict if they both use the same resource, and at most one of them uses it in read-only modality.

The definition of concurrent transitions is validated by the following lemma. 
Lemma 5 (Square lemma): If $\delta_{1}=M \stackrel{\eta_{1}}{\longrightarrow} N_{1}$ and $\delta_{2}=$ $M \stackrel{\eta_{2}}{\longrightarrow} N_{2}$ are two coinitial concurrent transitions, then there exist two cofinal transitions $\delta_{2} / \delta_{1}=N_{1} \stackrel{\eta_{2}}{\longrightarrow} N$ and $\delta_{1} / \delta_{2}=$ $N_{2} \stackrel{\eta_{1}}{\longrightarrow} N$.

Causal equivalence, denoted by $\asymp$, is the least equivalence relation between traces closed under composition that obeys the following rules:

$\delta_{1} ; \delta_{2} / \delta_{1} \asymp \delta_{2} ; \delta_{1} / \delta_{2} \quad \delta ; \delta_{\bullet} \asymp \epsilon_{\text {Source }(\delta)} \quad \delta_{\bullet} ; \delta \asymp \epsilon_{\operatorname{target}(\delta)}$

Intuitively causal equivalence identifies traces that differ only for swaps of concurrent actions and simplifications of inverse actions. Next result shows that there is a unique way to go from one state to another up to causal equivalence. This means that causal equivalent traces can be reversed in the same ways, and that traces which are not causal equivalent lead to distinct nets.

Theorem 1 (Causal consistency): Let $\theta_{1}$ and $\theta_{2}$ be coinitial traces, then $\theta_{1} \asymp \theta_{2}$ if and only if $\theta_{1}$ and $\theta_{2}$ are cofinal.

\section{CONTROLlED REVERSIBILITY}

In this section we define CR $\mu$ KLAIM, an extension of $\mu$ KLAIM featuring an explicit rollback facility to control $\mathrm{R} \mu$ KLAIM reversing capabilities, allowing us to program recovery activities inside $\mu$ KLAIM applications. We follow the general approach of [13], but adapted to deal with the interplay of the different $\mu$ KLAIM actions.

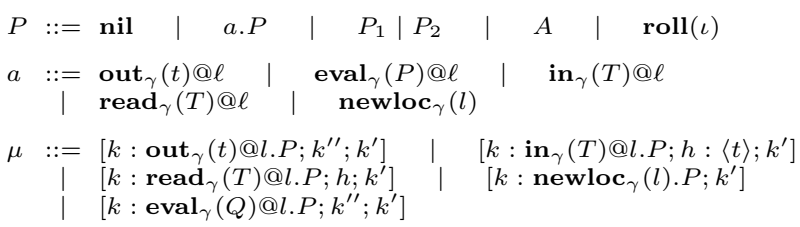

TABLE IX. $\quad$ CR $\mu$ KLAIM SYNTAX

CR $\mu$ KLAIM syntax extends R $\mu$ KLAIM syntax on two respects. First, actions in CR $\mu$ KLAIM are labeled by references $\gamma$, which act as variables for keys. Second, CR $\mu$ KLAIM introduces process $\operatorname{roll}(\gamma)$, which undoes the action labeled by $\gamma$. To simplify the technicalities, we change the syntax of memories as well, recording the continuation process also in out, eval, and newloc memories. Formally, we update the syntax of processes, actions and memories as reported in Table IX. Other syntactic categories are unchanged. At runtime references $\gamma$ are replaced by keys $k$, thus we use $\iota$ to range over both $\gamma$ and $k$. If $a_{\gamma}$ denotes an action labeled by $\gamma$, then $\gamma$ is bound in $a_{\gamma} . P$ with scope $P$. The definition of initial nets in $\mathrm{CR} \mu \mathrm{KLAIM}$ is extended w.r.t. Definition 1 , by also requiring that they do not contain any $\operatorname{roll}(k)$ (the argument of roll is always a reference), nor free occurrences of references. Well-formedness changes accordingly. Structural congruence coincides with the one of R $\mu$ KLAIM. For simplicity we denote memories as $[k: a . P ; \xi]$, where $a$ is one of the CR $\mu \mathrm{KLAIM}$ actions and $\xi$ is the additional information (e.g., the remaining keys in an out memory, the read tuple and the continuation key in an in memory, and so on). For readability's sake we omit references when they are not relevant.

The following result will help us in the definition of CR $\mu$ KLAIM semantics.
Lemma 6 (Net normal form): For any $\mathrm{CR} \mu \mathrm{KLAIM}$ net $N$, we have:

$$
\begin{aligned}
N \equiv(\nu \tilde{z}) \|_{l \in L}(l:: & \prod_{i \in I}\left(k_{i}: P_{i}\right)\left|\prod_{j \in J}\left[k_{j}: a_{j} . P_{j} ; \xi_{j}\right]\right| \\
& \prod_{h \in H}\left(k_{h} \prec\left(k_{h}^{2}, k_{h}^{3}\right)\right)\left|\prod_{x \in X}\left(k_{x}:\left\langle e t_{x}\right\rangle\right)\right| \\
& \prod_{w \in W}\left[k_{w}^{1}: \operatorname{in}_{\gamma_{w}}\left(T_{w}\right) @ l_{w} \cdot P_{w} ; k_{w}^{2}:\left\langle t_{w}\right\rangle ; k_{w}^{3}\right] \mid \\
& \left.\prod_{y \in Y}\left[k_{y}^{1}: \operatorname{read}_{\gamma_{y}}\left(T_{y}\right) @ l_{y} \cdot P_{y} ; k_{y}^{2} ; k_{y}^{3}\right]\right)
\end{aligned}
$$

where action $a_{j}$ is neither in nor read for every $j \in J$.

Definition 5 (CR $\mu$ KLAIM semantics): The $\quad \mathrm{CR} \mu \mathrm{KLAIM}$ operational semantics consists of a forward reduction relation $\mapsto_{c}$ and a backward reduction relation $\rightsquigarrow_{c}$. The backward reduction relation is the smallest evaluation-closed relation satisfying rule (Roll) in Table $\mathrm{X}$. The forward reduction relation is the smallest evaluation-closed relation satisfying the forward rules in Table $X$. These rules are the forward rules of Table VII extended to instantiate $\gamma$ with the proper key.

Backward reductions in CR $\mu$ KLAIM correspond to executions of the roll operator. Since all the occurrences of references $\gamma$ are bound, when a roll becomes enabled its argument is always a key $k$, uniquely identifying the memory created by the action to be undone. Thus, backward reductions are defined by the semantics of $\operatorname{roll}(k)$. The semantics involves many subtleties, related to the behavior of the different actions. However, we define just one rule, (Roll), capturing all of them.

The $\operatorname{roll}(k)$ operator should undo all the actions depending on the target action $k$, and only them. The all part is captured by the notion of completeness (Definition 3), and the only part by a notion of $k$-dependence (written $<$ :) defined below. The term $M$ in rule (Roll) captures the part of the net involved in the reduction. As a result of the reduction, $M$ disappears, leaving just the process $k: a . P$ that was inside the memory. If the action $a$ was an in, then also the consumed tuple should be restored. This is the role of $N_{t}$. Also, unless the locality containing the roll has been created by a descendant of $k$, it has to be preserved. This is the role of $N_{l}$. Finally, resources taken by the computation from the context should be given back to the context. This is the role of $N \xi_{k}$ (see Definition 8).

We now define formally the notations used in the definition of the semantics. Causal dependence among keys and localities is needed for $k$-dependence.

Definition 6 (Causal dependence): Let $N$ be a CR $\mu$ KLAIM net and $T_{N}$ the set of keys and localities in $N$. The relation $<:_{N}$ on $T_{N}$ is the smallest preorder (i.e., reflexive and transitive relation) satisfying:

- $\quad k<:_{N} k^{\prime}$ if one of $\left[k: \operatorname{out}_{\gamma}(t) @ l . P ; k_{1} ; k_{2}\right]$, $\left[k: \operatorname{eval}_{\gamma}(Q) @ l . P ; k_{1} ; k_{2}\right], k \prec\left(k_{1}, k_{2}\right)$ occurs in $N$, with $k^{\prime}=k_{1}$ or $k^{\prime}=k_{2}$;

- $\quad k<:_{N} k^{\prime}$ if one of $\left[k_{1}: \operatorname{in}_{\gamma}(T) @ l . P ; k_{2}:\langle e t\rangle ; k^{\prime}\right]$, $\left[k_{1}: \operatorname{read}_{\gamma}(T) @ l . P ; k_{2} ; k^{\prime}\right]$ occurs in $N$, with $k=k_{1}$ or $k=k_{2}$; 


\begin{tabular}{|c|}
\hline $\begin{array}{c}M=(\nu \tilde{z}) l:: k^{\prime}: \operatorname{roll}(k)\left\|l^{\prime}::[k: a \cdot P ; \xi]\right\| N \\
N_{t}=l^{\prime \prime}:: h:\langle t\rangle \text { if } a=\operatorname{in}_{\gamma}(T) @ l^{\prime \prime} \wedge \xi=h:\langle t\rangle ; k^{\prime \prime}, \text { otherwise } N_{t}=\mathbf{0}\end{array}$ \\
\hline$(\nu \tilde{z}) l:: k^{\prime}: \operatorname{roll}(k)\left\|l^{\prime}::[k: a . P ; \xi]\right\| N \rightsquigarrow_{c} l^{\prime}:: k: a . P\left\|N_{t}\right\| N_{l} \| N_{\text {夕 } k}$ \\
\hline$\llbracket t \rrbracket=e t$ \\
\hline$\overline{l:: k: \text { out }_{\gamma}(t) @ l^{\prime} . P \| l^{\prime}:: \text { empty } \mapsto_{c}\left(\nu k^{\prime}, k^{\prime \prime}\right)\left(l:: k^{\prime}: P[k / \gamma] \mid\left[k: \operatorname{out}_{\gamma}(t) @ l^{\prime} . P ; k^{\prime \prime} ; k^{\prime}\right] \| l^{\prime}:: k^{\prime \prime}:\langle e t\rangle\right)}$ (Out) \\
\hline $\operatorname{match}(\llbracket T \rrbracket, e t)=\sigma$ \\
\hline$l:: k: \mathbf{i n}_{\gamma}(T) @ l^{\prime} . P\left\|l^{\prime}:: h:\langle e t\rangle \mapsto_{c}\left(\nu k^{\prime}\right)\left(l:: k^{\prime}: P[k / \gamma] \sigma \mid\left[k: \mathbf{i n}_{\gamma}(T) @ l^{\prime} . P ; h:\langle e t\rangle ; k^{\prime}\right]\right)\right\| l^{\prime}::$ empty \\
\hline \multirow[t]{2}{*}{$\operatorname{match}(\llbracket T \rrbracket, e t)=\sigma$} \\
\hline \\
\hline$l:: k:$ newloc $_{\gamma}\left(l^{\prime}\right) \cdot P \mapsto_{c}\left(\nu l^{\prime}\right)\left(\left(\nu k^{\prime}\right)\left(l:: k^{\prime}: P[k / \gamma] \mid\left[k:\right.\right.\right.$ newloc $\left.\left._{\gamma}\left(l^{\prime}\right) \cdot P ; k^{\prime}\right]\right) \| l^{\prime}::$ empty $) \quad(N e w)$ \\
\hline$l:: k: \mathbf{e v a l}_{\gamma}(Q) @ l^{\prime} . P \| l^{\prime}:: \mathbf{e m p t y} \mapsto_{c}\left(\nu k^{\prime}, k^{\prime \prime}\right)\left(l:: k^{\prime}: P[k / \gamma] \mid\left[k: \operatorname{eval}_{\gamma}(Q) @ l^{\prime} . P ; k^{\prime \prime} ; k^{\prime}\right] \| l^{\prime}:: k^{\prime \prime}: Q\right)$ \\
\hline
\end{tabular}

- $\quad k<:_{N} z$ if $\left[k:\right.$ newloc $\left._{\gamma}(l) . P ; k^{\prime}\right]$ occurs in $N$, with $z=l$ or $z=k^{\prime}$;

- $\quad l<:_{N} k$ if $l:: k: P$ or $l:: k:\langle e t\rangle$ occurs in $N$.

Note that for action out the continuation and the tuple depend on the action, while for actions in and read the continuation depends on both the action and the tuple. The last clause specifies that tuples and processes depend on the locality where they are. We can now define $k$-dependence.

Definition 7 ( $k$-dependence): Let $N$ be a CR $\mu$ KLAIM net in normal form (see Lemma 6). Net $N$ is $k$-dependent, written $k<: N$, if: (i) for every $i \in I \cup J \cup H \cup X$ we have $k<:_{N} k_{i}$; (ii) for every $i \in W \cup Y$ we have $k<:_{N} k_{i}^{1}$ or $k<:_{N} k_{i}^{2}$; and (iii) for every $z \in \tilde{z}$ we have $k<:_{N} z$.

We now describe the resources taken from the environment that need to be restored. We start with a simple example.

Example 2: Consider the following net:

$$
l:: k: \operatorname{out}_{\gamma}(\text { foo }) @ l \cdot \operatorname{in}(f \circ o 1) @ l \cdot \operatorname{roll}(\gamma) \mid k^{\prime}:\langle f o o 1\rangle
$$

After two steps the net becomes:

$$
\begin{aligned}
\left(\nu k^{\prime \prime}, k^{\prime \prime \prime}, k^{\prime \prime \prime \prime}\right)\left(l:: k^{\prime \prime \prime \prime}: \operatorname{roll}(k) \mid k^{\prime \prime \prime}:\langle f o o\rangle\right. \\
\mid\left[k: \operatorname{out}_{\gamma}(\text { foo }) @ l \cdot \operatorname{in}(f \circ o 1) @ l \cdot \operatorname{roll}(\gamma) ; k^{\prime \prime \prime} ; k^{\prime \prime}\right] \\
\left.\mid\left[k^{\prime \prime}: \operatorname{in}(\text { foo } 1) @ l \cdot \operatorname{roll}(k) ; k^{\prime}:\langle\text { foo } 1\rangle ; k^{\prime \prime \prime \prime}\right]\right)
\end{aligned}
$$

Performing $\operatorname{roll}(k)$ should lead back to the initial state. Releasing only the content of the target memory is not enough, since also the tuple $k^{\prime}:\langle f o o 1\rangle$ should be released. This tuple is restored by $N \xi_{k}$ in rule (Roll), since it is in a memory in $N$, but $k^{\prime}$ does not depend on $k$.

Projection, defined below, should release the tuples consumed by in actions which are undone, and also in and read actions that accessed a tuple created by an out action that is undone. Resources are released only if they do not depend on the key $k$ of the roll.
Definition 8 (Projection): Let $N$ be a net in normal form (see Lemma 6). If $k \notin \tilde{z}$ then:

$$
\begin{gathered}
N \xi_{k} \equiv(\nu \tilde{z}) \|_{l \in L^{\prime}}\left(l:: \prod_{w \in W^{\prime}} k_{w}^{1}: \operatorname{in}_{\gamma_{w}}\left(T_{w}\right) @ l_{w} \cdot P_{w}\right. \\
\left.\prod_{y \in Y^{\prime}} k_{y}^{1}: \operatorname{read}_{\gamma_{y}}\left(T_{y}\right) @ l_{y} \cdot P_{y}\right) \\
\|_{w \in W^{\prime \prime}}\left(l_{w}:: k_{w}^{2}:\left\langle t_{w}\right\rangle\right)
\end{gathered}
$$

where $L^{\prime}=\left\{l \in L \mid k \nless:_{N} l\right\}, W^{\prime}=\left\{w \in W \mid k \nless:_{N} k_{w}^{1}\right\}$, $Y^{\prime}=\left\{y \in Y \mid k \nless:_{N} k_{y}^{1}\right\}$ and $W^{\prime \prime}=\left\{w \in W \mid k \nless:_{N} k_{w}^{2}\right\}$.

We show now that CR $\mu$ KLAIM is indeed a controlled version of $\mathrm{R} \mu \mathrm{KLAIM}$. Let erCon be a function from CR $\mu$ KLAIM nets to $\mathrm{R} \mu \mathrm{KLAIM}$ nets which is the identity but for replacing $\operatorname{roll}(\iota)$ with nil, and removing continuations inside memories for out, eval and newloc, and references $\gamma$.

Theorem 2: Given a CR $\mu \mathrm{KLAIM}$ net $N$, if $N \mapsto_{c} M$ then $\operatorname{erCon}(N) \mapsto_{r} \operatorname{erCon}(M)$ and if $N \rightsquigarrow_{c} M$ then $\operatorname{erCon}(N) \rightsquigarrow_{r}^{+} \operatorname{erCon}(M)$ where $\rightsquigarrow_{r}^{+}$is the transitive closure of $\rightsquigarrow_{r}$.

\section{A FRANCHISING SCENARIO}

In this section, we apply our reversible languages to a simplified but realistic franchising scenario, where a number of franchisees affiliate to a franchisor and determine the price of goods to expose to their customers. Each franchisee obtains a lot of goods from the market, gets the suggested price from the corresponding franchisor, possibly modifies it according to some local policy, and then publishes the computed price. In case of errors, e.g., the computed price is not competitive, franchisees can change price and, possibly, franchisor by undoing and performing again the activities described above. Notably, this does not affect the franchisors and the other franchisees. Instead, when a franchisor needs to change the suggested 


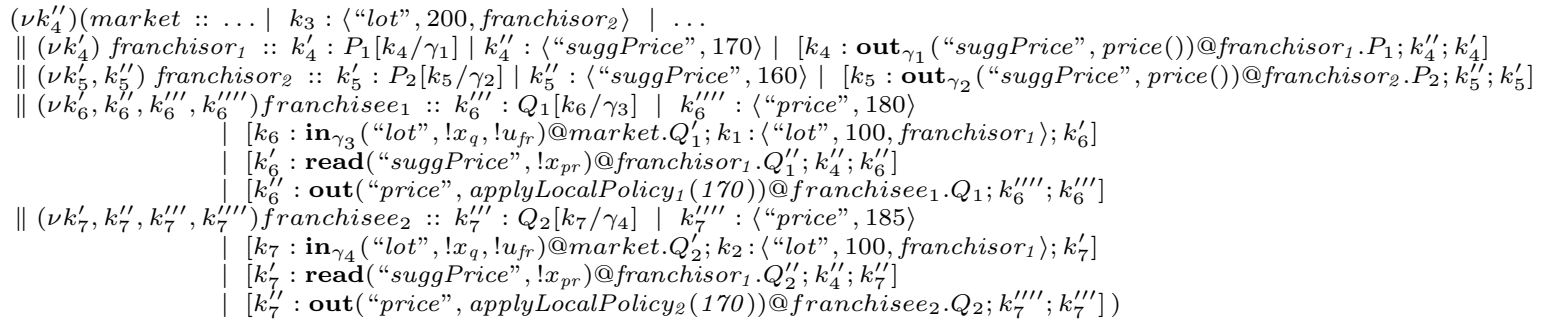

TABLE XII. CR $\mu$ KLAIM NET OF THE FRANCHISING SCENARIO (AFTER A FEW FORWARD STEPS)

price, it performs a backward computation that involves all the affiliated franchisees. For the sake of presentation, hereafter we consider a scenario consisting of the market, two franchisors and two franchisees.

The whole scenario is rendered in CR $\mu$ KLAIM as the net in Table XI, where:

$$
\begin{aligned}
& P_{1}=\text { in("chgPr")@ } \text { franchisor }_{1} \text {.roll }\left(\gamma_{1}\right) \\
& P_{2}=\operatorname{in}\left(\text { "chgPr")@ } \text { franchisor }_{2} \cdot \operatorname{roll}\left(\gamma_{2}\right)\right. \\
& Q_{1}=\operatorname{in}(" c h g P r ") @ \text { franchisee }_{1} \cdot \operatorname{roll}\left(\gamma_{3}\right) \\
& Q_{2}=\text { in("chgPr")@ franchisee } 2 . \operatorname{roll}\left(\gamma_{4}\right)
\end{aligned}
$$

The market is a storage of tuples, representing lots of goods, of the form 〈"lot", $v, l\rangle$, where $v$ indicates a number of items and $l$ the locality of the franchisor providing the lot. Each franchisor is a node executing a process that produces the suggested price, by resorting to a (non specified) function price(). Then, it waits for a change price request (i.e., a tuple ("chgPr") ) to trigger the rollback of the executed activity (by means of the roll operator). Such tuple could be generated by a local process monitoring the selling trend that we leave unspecified and omit. The franchisees are nodes executing processes with the same structure. Each of them first gets a lot from the market, by consuming a lot tuple. Then, it reads the suggested price from the corresponding franchisor and uses it to determine the local price (using the unspecified function applyLocalPolicy $(\cdot))$. Finally, similarly to the franchisor process, it waits for a change price request and possibly rolls back.

Consider a net evolution where the two franchisors produce their suggested prices (170 and 160 cents, respectively) and the two franchisees acquire the first two lots, read the suggested price and publish their local prices (by increasing the suggested price by 10 and 15 cents, respectively). The resulting net is shown in Table XII, where $Q_{i}^{\prime}$ and $Q_{i}^{\prime \prime}$ denote the continuations of the in and read actions.

If franchisee $_{1}$ needs to change its lot of goods, a tuple $\langle$ "chgPr" $\rangle$ is locally produced and, then, the rollback is triggered by $\operatorname{roll}\left(k_{6}\right)$. In this way, the memory of the first in will be directly restored (and its forward history deleted), rather than undoing action-by-action the forward execution (as in $\mathrm{R} \mu \mathrm{K}$ LAIM). As expected, the backward step does not affect franchisee $_{2}$. Instead, if franchisor $_{1}$ wants to change the price stored in $k_{4}^{\prime \prime}$ : 〈"suggPrice", 170〉, it undoes action $k_{4}$ : out("suggPrice", price())@ franchisor ${ }_{1}$ by also involving the read memories within franchise $e_{1}$ and franchisee 2 , because $k_{4}^{\prime \prime}$ occurs within them, and all the occurrences of $k_{4}^{\prime \prime}$ must be considered to apply rule (Roll). Thus, the projection operation $\cdot \sum_{k_{4}}$ will restore the read actions from their memories. This allows the franchisees affiliated to this franchisor to adjust their local prices.

If we replace the actions read("suggPrice", ! $\left.x_{p r}\right) @ u_{f r}$ by in("suggPrice", ! $\left.x_{p r}\right) @ u_{f r}$. out("suggPrice", $\left.x_{p r}\right) @ u_{f r}$, the (undesired) side effect already discussed in the simple example shown in Section III arises: if a franchisee changes its lot, it may involve in the undo procedure the other franchisees.

Notably, by setting processes $P_{i}$ and $Q_{i}$ to nil and removing all references, we obtain a $\mathrm{R} \mu \mathrm{KLAIM}$ specification, which exhibits computations as the one above (Theorem 2), but also other computations mixing forward and backward actions in an uncontrolled way that are undesired in this scenario.

\section{RELATED WORK AND CONCLUSION}

The history of reversibility in a sequential setting is already quite long [16], [7]. Our work however concerns causalconsistent reversibility, which has been introduced in [5]. This work considered causal-consistent reversibility for CCS, introducing histories for threads to track causality information. A generalization of the approach, based on the transformation of dynamic operators into static, has been proposed in [18]. Both the works are in the setting of uncontrolled reversibility, and they consider labeled semantics. Labeled semantics for uncontrolled reversibility has been also studied for $\pi$ calculus [4], while reduction semantics has been studied for $\mathrm{HO} \pi$ [14] and $\mu \mathrm{Oz}$ [17]. We are closer to [14], which uses modular memories similar to ours. Controlled reversibility has been studied first in [6], introducing irreversible actions, then in [1], where energy parameters drive the evolution of the process, and in [19], where a non-reversible controller drives a reversible process. For an exhaustive survey on causalconsistent reversibility we refer to [15].

The main novelty of our work concerns the analysis of the interplay between reversibility on the one hand, and tuplebased communication on the other hand. The results we discussed correspond to some of the results in [14], [13], which were obtained in the simpler framework of $\mathrm{HO} \pi$. 
We have not yet transported to $\mu$ KLAIM all the results in [14], [13], [12]. The main missing results are an encoding from the reversible calculus into the basic one [14], a more low level semantics for controlled reversibility [13], and the introduction of alternatives to avoid repeating the same error after a rollback [12]. A full porting of the results above would need to study the behavioral theory of $\mathrm{R} \mu \mathrm{KLAIM}$ and $\mathrm{CR} \mu \mathrm{KLAIM}$, which is left for future work. We outline however below how the issues above can be faced.

The most natural way to add alternatives [12] to $\mathrm{CR} \mu \mathrm{KLAIM}$ is to attach them to tuples. For instance, $k$ : $\langle f o o\rangle \%\langle f o o 1\rangle$ would mean "try $\langle f o o\rangle$, then try $\langle f o o 1\rangle$ ". Such a tuple behaves as $k:\langle f o o\rangle$, but it becomes $k:\langle f o o 1\rangle$ when it is inside a memory of an in, and a roll targeting the memory is executed. As in $\mathrm{HO} \pi$, such a simple mechanism considerably increases the expressiveness.

A faithful encoding of $\mathrm{R} \mu \mathrm{KLAIM}$ and $\mathrm{CR} \mu \mathrm{KLAIM}$ into $\mu \mathrm{KLAIM}$ itself would follow the lines of [14]. Its definition would be simpler than that for reversible $\mathrm{HO} \pi$ [14], since tuple spaces provide a natural storage for memories and connectors. Such encoding will pave the way to the use of KLAVA [3], a framework providing run-time support for KLAIM actions in Java, to experiment with reversible distributed applications.

A low-level semantics for CR $\mu$ KLAIM, more suitable to an implementation, should follow the idea of [13], based on an exploration of the causal dependences of the memory pointed by the roll. However, one has to deal with read dependences, and at this more concrete level the use of restriction is no more viable. Thus, one should keep in each tuple the keys of processes that have read it.

\section{ACKNOWLEDGMENT}

The authors gratefully thank the anonymous referees for their useful remarks. This work was partially supported by Italian MIUR PRIN Project CINA Prot. 2010LHT4KM and by the French ANR project REVER n. ANR 11 INSE 007.

\section{REFERENCES}

[1] G. Bacci, V. Danos, and O. Kammar. On the Statistical Thermodynamics of Reversible Communicating Processes. In $C A L C O$, volume 6859 of $L N C S$, pages 1-18. Springer, 2011.
[2] L. Bettini, V. Bono, R. De Nicola, G. L. Ferrari, D. Gorla, M. Loreti, E. Moggi, R. Pugliese, E. Tuosto, and B. Venneri. The Klaim Project: Theory and Practice. In Global Computing, volume 2874 of LNCS, pages $88-150$. Springer, 2003.

[3] L. Bettini, R. De Nicola, and R. Pugliese. Klava: a Java Package for Distributed and Mobile Applications. Software - Pract. Exper., 32(14):1365-1394, 2002.

[4] I. D. Cristescu, J. Krivine, and D. Varacca. A Compositional Semantics for the Reversible Pi-calculus. In LICS, pages 388-397. IEEE Press, 2013.

[5] V. Danos and J. Krivine. Reversible Communicating Systems. In CONCUR, volume 3170 of LNCS, pages 292-307. Springer, 2004.

[6] V. Danos and J. Krivine. Transactions in RCCS. In CONCUR, volume 3653 of $L N C S$, pages 398-412. Springer, 2005.

[7] V. Danos and L. Regnier. Reversible, Irreversible and Optimal LambdaMachines. Theor. Comput. Sci., 227(1-2), 1999.

[8] R. De Nicola, G. Ferrari, and R. Pugliese. KLAIM: A Kernel Language for Agents Interaction and Mobility. T. Software Eng., 24(5):315-330, 1998.

[9] D. Gelernter. Generative Communication in Linda. ToPLaS, 7(1):80112, 1985.

[10] E. Giachino, I. Lanese, C. A. Mezzina, and F. Tiezzi. Causal-Consistent Reversibility in a Tuple-Based Language (TR). http://www.cs.unibo.it/ $\sim$ lanese/work/klaimrev-TR.pdf.

[11] D. Gorla and R. Pugliese. Resource Access and Mobility Control with Dynamic Privileges Acquisition. In ICALP, volume 2719 of LNCS, pages 119-132. Springer, 2003.

[12] I. Lanese, M. Lienhardt, C. A. Mezzina, A. Schmitt, and J.-B. Stefani. Concurrent Flexible Reversibility. In ESOP, volume 7792 of LNCS, pages 370-390. Springer, 2013.

[13] I. Lanese, C. A. Mezzina, A. Schmitt, and J.-B. Stefani. Controlling Reversibility in Higher-Order Pi. In CONCUR, volume 6901 of $L N C S$, pages 297-311. Springer, 2011.

[14] I. Lanese, C. A. Mezzina, and J.-B. Stefani. Reversing Higher-Order Pi In CONCUR, volume 6269 of LNCS, pages 478-493. Springer, 2010.

[15] I. Lanese, C. A. Mezzina, and F. Tiezzi. Causal-consistent reversibility. Bulletin of the EATCS, 114, 2014.

[16] G. Leeman. A Formal Approach to Undo Operations in Programming Languages. ToPLaS, 8(1), 1986.

[17] M. Lienhardt, I. Lanese, C. A. Mezzina, and J.-B. Stefani. A Reversible Abstract Machine and Its Space Overhead. In FMOODS/FORTE, volume 7273 of $L N C S$, pages 1-17. Springer, 2012.

[18] I. Phillips and I. Ulidowski. Reversing Algebraic Process Calculi. J. Log. Algebr. Program., 73(1-2):70-96, 2007.

[19] I. Phillips, I. Ulidowski, and S. Yuen. A Reversible Process Calculus and the Modelling of the ERK Signalling Pathway. In $R C$, volume 7581 of LNCS, pages 218-232. Springer, 2012. 